\title{
Late Onset Tay-Sachs Disease in a Non-Jewish Patient: Case Report
}

\author{
Smaranda Maier ${ }^{1}$, Zoltan Bajko1*, Anca Moțățăianu1 ${ }^{1}$, Adina Stoian², Bianca Șchiopu³, Rodica Bălașa1 \\ 1. University of Medicine and Pharmacy Targu Mures, Department of Neurology \\ 2. University of Medicine and Pharmacy Targu Mures, Department of Patophysiology \\ 3. Mureș County Clinical Emergency Hospital, Târgu Mureș
}

Tay-Sachs disease (TSD) is a rare, inherited, autosomal rececessive lysosomal storage disease. The late-onset form is an uncommon condition among non-Jewish population.

We present the case of a 32 years old male patient without Jewish origins, in whom the disease began in adolescence and was initially diagnosed with spinal muscular atrophy. He developed progressively protean neurological symptomatology, including tetraparesis, cerebellar and extrapyramidal syndromes. The diagnosis was based on the cerebral MRI, showing severe cerebellar atrophy and the determination of the Hexosaminidase A activity, revealing low level.

In patients showing signs of lower motor neuron involvement, cerebellar and pyramidal signs and marked cerebellar atrophy the late-onset TSD should be suspected, and the first step in establishing the diagnosis should be to determine the serum activity of Hexosaminidase A.

Keywords: Tay-Sachs disease, autosomal rececessive, hexosaminidase A

Received 14 August 2017 / Accepted 31 October 2017

\section{Introduction}

Tay-Sachs disease (TSD) is the most frequent form of the GM2-gangliosidoses, a heterogenous group of lysosomal storage diseases that are inherited in an autosomal rececessive manner. Hexosaminidase (Hex) A is a lysosomal enzyme, which degrades the ganglioside GM2, and is composed of an alfa subunit encoded by the HEXA gene and a beta subunit encoded by the HEXB gene. In TSD, a mutation in the HEXA gene, causes a deficiency of HexA, resulting in an accumulation of GM2 gangliosides in the nervous system. (1)

TSD has two clinical forms of evolution. The most common form is the infantile or acute TSD, with severe reduction of HexA activity (less than $0.5 \%$ ), characterized by a rapid evolution, leading to death between the ages of 3 and 5 years. The second form, late-onset TSD is associated with a higher HexA activity of $2-5 \%$ of normal levels and has a more prolonged clinical course. It is, in turn, subdivided into juvenile or sub-acute form which usually leads to a vegetative state before the age of 15 and adult or chronic form, with onset in late childhood, in adolescence, or even later. Residual enzyme activity of over $10 \%$ does not cause clinical manifestations. $(2,3,4,5)$

TSD has an increased prevalence in the Ashkenazi Jewish population, even if the introduction of carrier screening programs and prenatal diagnosis has led to a decrease in the incidence of TDS with approximately $90 \%$ in the Jewish population. (1)

Late-onset TSD is considered to be a rare condition among people without Ashkenazi Jewish ancestry. $(2,6)$
We present the case of a patient without Jewish origins, in whom the disease began in adolescence and was initially diagnosed with spinal muscular atrophy.

\section{Case report:}

A 32 years old male patient, with negative medical and family history, without consanguinity, developed after the age of 17 an inconstant clumsiness and mild tremor in the upper extremities. At the age of 19 he developed a predominant proximal muscle weakness in the lower extremities, with slowly progressive aggravation, later he developed muscle atrophyes and fasciculation in the thigh and shoulder girdle muscles. At the age of 25 he presented swallowig difficulties, dysartria, coordination and equilibrium problems. He was diagnosed initially with spinal muscular atrophy.

At the age of 32 he was addressed to our department with the suspicion of amyotrophic lateral sclerosis. The neurological examination at the admission revealed bilateral horizontal nystagmus, without gaze abnormalities, diminished pharyngeal reflexes, dysphagia for solid foods, paraparetic and ataxic gait, tetraparesis with grade 4 motor deficit in the upper extremities, grade 3 in the proximal muscle groups and grade 4 in the distal muscle groups of the lower limbs, exaggerated deep tendon reflexes in the upper extremities, diminished deep tendon reflexes in the lower extremities, bilateral plantar clonus, flexor plantar response, right sided Rossner sign, positive palmomental reflex, cogwheel sign in the upper extremities, postural tremor of the extremities, truncal ataxia, bilateral dysmetria, generalysed muscle atrophies, fasciculations in the thigh and shoulder girdle muscles, dysartria, normal cognition. 
The laboratory examinations (complete blood count, biochemistry, Erithrocyte Sedimentation Rate, serum protein immunoelectrophoresis, autoimmune markers, VDRL, thyroid hormones) were in normal range.

The cerebral and spinal cord magnetic resonance imaging exam was normal, excepting a severe cerebellar atrophy. (Figure 1, 2)

The electrodiagnostic studies show normal motor and sensory nerve conduction parameters. The needle EMG examination shows chronic neurogenic changes in the lumbar and cervical myotomes. (Figure 3.) The electrophysiologic findings are consistent with chronic generalized disorder of the motor neurons, their axons, or both, in a context of a a slowly progressive degenerative disorder.

The psychological workup revealed normal cognition,
MMSE 30 p, normal clock-drawing test result (10/10), without psychiatric symptoms.

The ophtalmological examination revealed normal conditions.

The HexA and HexB activities were measured, the HexA activity was low $(0.02 \mathrm{nmol} / \mathrm{spot}$, normal values: 0.6-2.4 $\mathrm{nmol} / \mathrm{spot}$ ).

The diagnosis of GM2 gangliosidosis, adult onset Tay-Sachs disease was established. The genetical analysis was not possible.

\section{Discussions}

Late-onset TSD is an extremely rare condition among non-Jewish people, its real incidence is unknown, but is considered to be somewhere around 1 case per 300,000

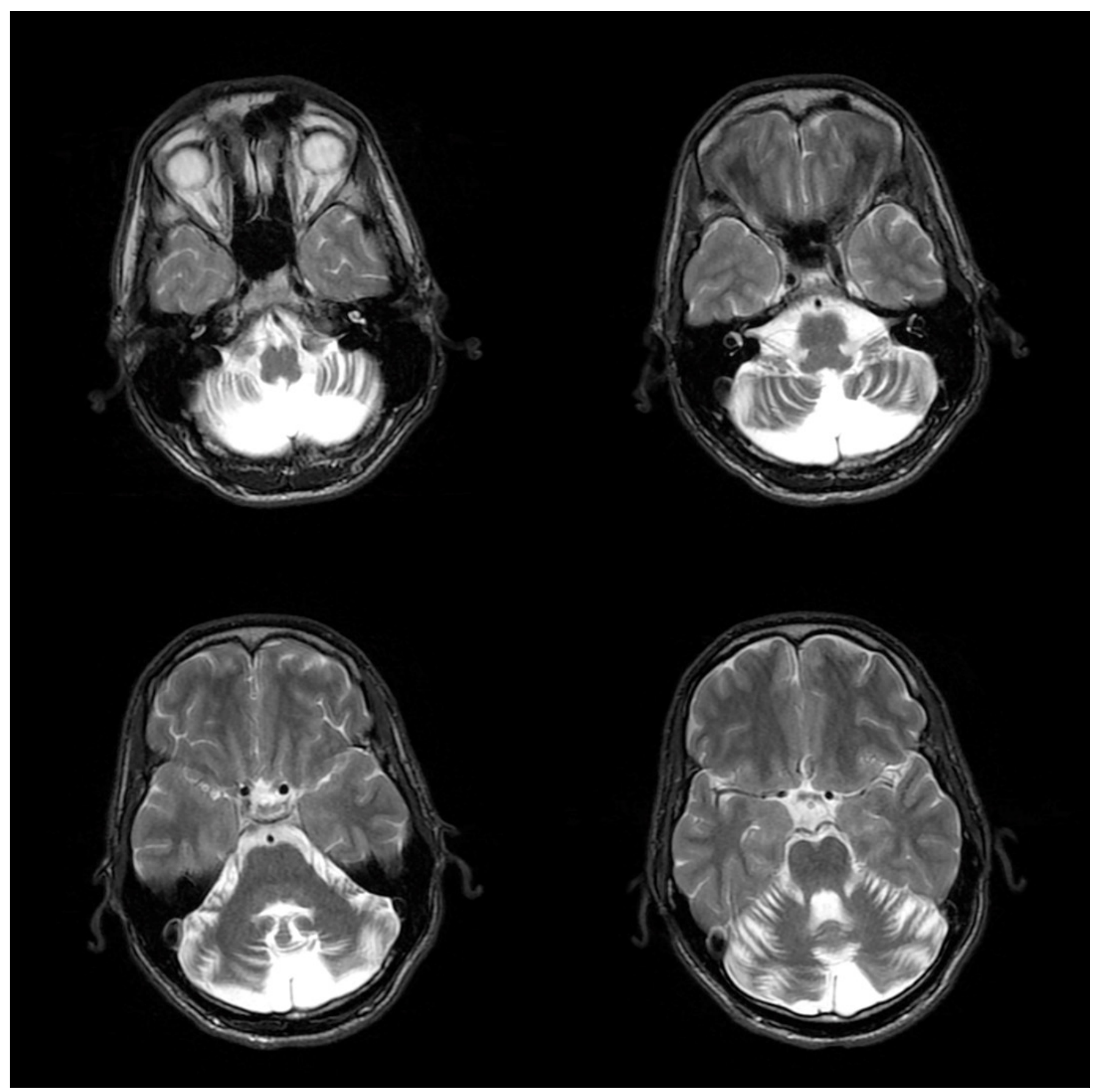

Fig. 1. Axial T2 weighted cerebral MRI revealing severe cerebellar atrophy 


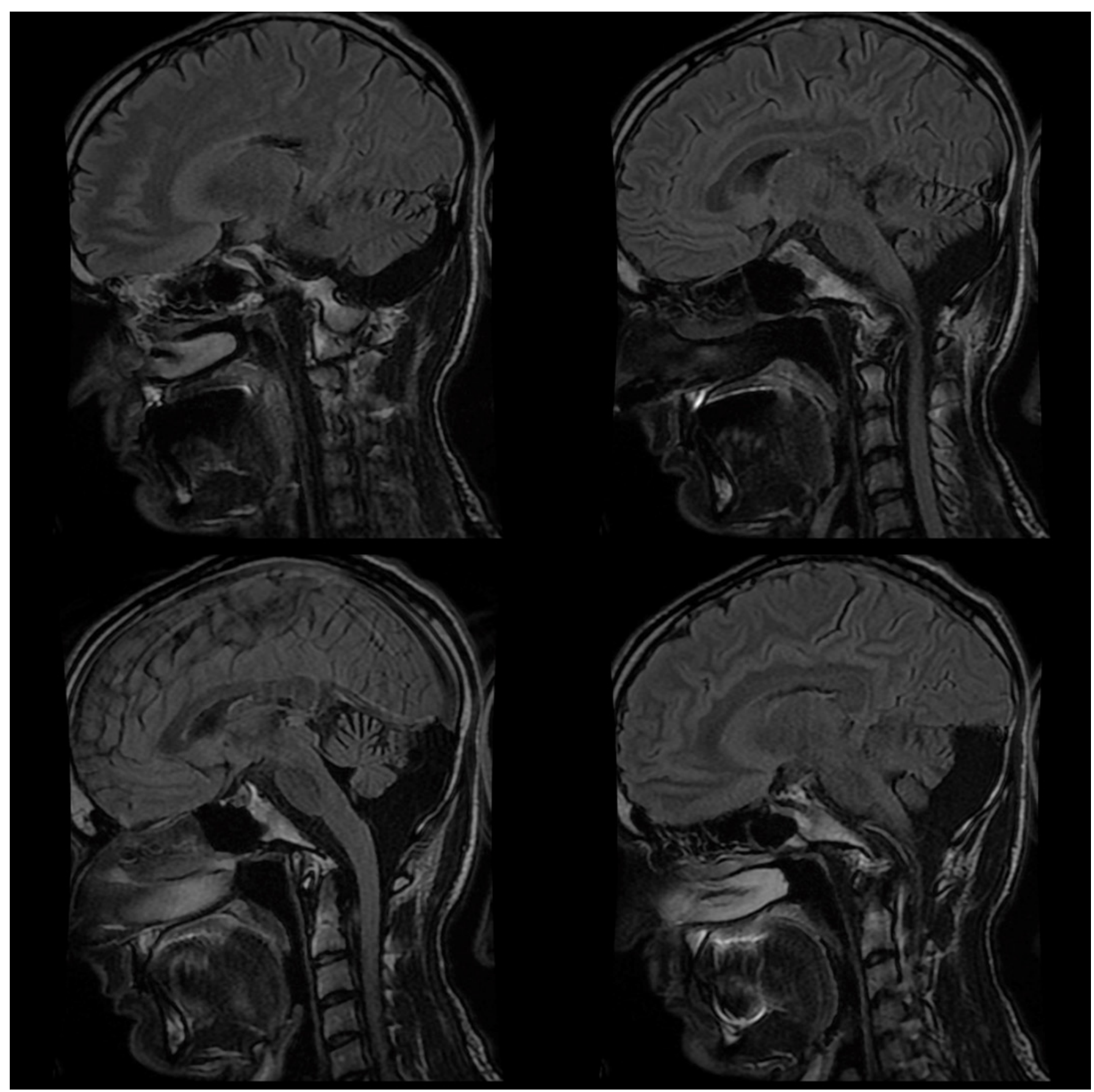

Fig. 2. Sagittal T2 FLAIR weighted cerebral MRI revealing severe cerebellar atrophy

people. In the Ashkenazi Jewish people its incidence is 1 case per 135,000 people. (2)

In patients with late-onset TSD, HexA activity is decreased between $2-5 \%$ of normal values, thus causing a late onset and a slower evolution in deterioration of motor, cerebellar and spinocerebellar functions.(3) In some patients, the disease begins in adolescence with cerebelar (dysarthria, ataxia, tremor, dysmetria) or motor signs (proximal motor weakness, atrophies, fasciculations) and in others the predominant symptoms at onset are those of a psychiatric nature (psychosis, recurrent depression, bipolar disorders). (2) In the case of our patient, psychiatric symptoms are completely absent, the disease being manifested at onset by cerebellar and motor signs.
A delay in the diagnosis of late-onset TSD of 15 years in our case, was also found by Neudorfer $\mathrm{O}$ et al. They performed a study on 21 patients with late-onset TSD and observed that 17 patients were correctly diagnosed late, about 8-10 years after the onset of the symptoms, the initial diagnosis being of muscular spinal atrophy, amyotrophic lateral sclerosis or spinocerebellar ataxia.(2) Our patient was initially diagnosed with spinal muscular atrophy.

Considering the predominance of the proximal motor deficit at the onset of the disease, besides the diagnosis of spinal muscular atrophy, GM2 gangliosidoses should also be considered in the case of our patient, and dosing of the HexA enzyme would have been an adequate step for establishing earlier the right diagnosis. (7-9) 


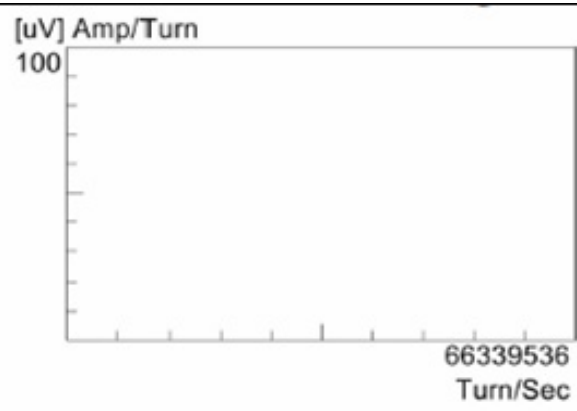

\begin{tabular}{|c|c|c|c|c|c|c|c|}
\hline \multicolumn{8}{|l|}{ MUP Summary } \\
\hline \multicolumn{5}{|l|}{ Range } & \multirow{2}{*}{\begin{tabular}{|l} 
Dur. \\
$10.6 \mathrm{~ms}$
\end{tabular}} & \multirow{2}{*}{\begin{tabular}{|l} 
Amp. \\
$1891.2 \mathrm{uV}$
\end{tabular}} & \multirow{2}{*}{$\begin{array}{l}\text { Phases } \\
7.5\end{array}$} \\
\hline Polyphasic MUPs & 6 & of 6 & $(100$ & s) & & & \\
\hline Remaining MUPs & 0 & of 6 & $(0$ & s) & & & \\
\hline All MUPs & 6 & & & & $10.6 \mathrm{~ms}$ & 1891.2uV & 7.5 \\
\hline
\end{tabular}

MUP Analysis

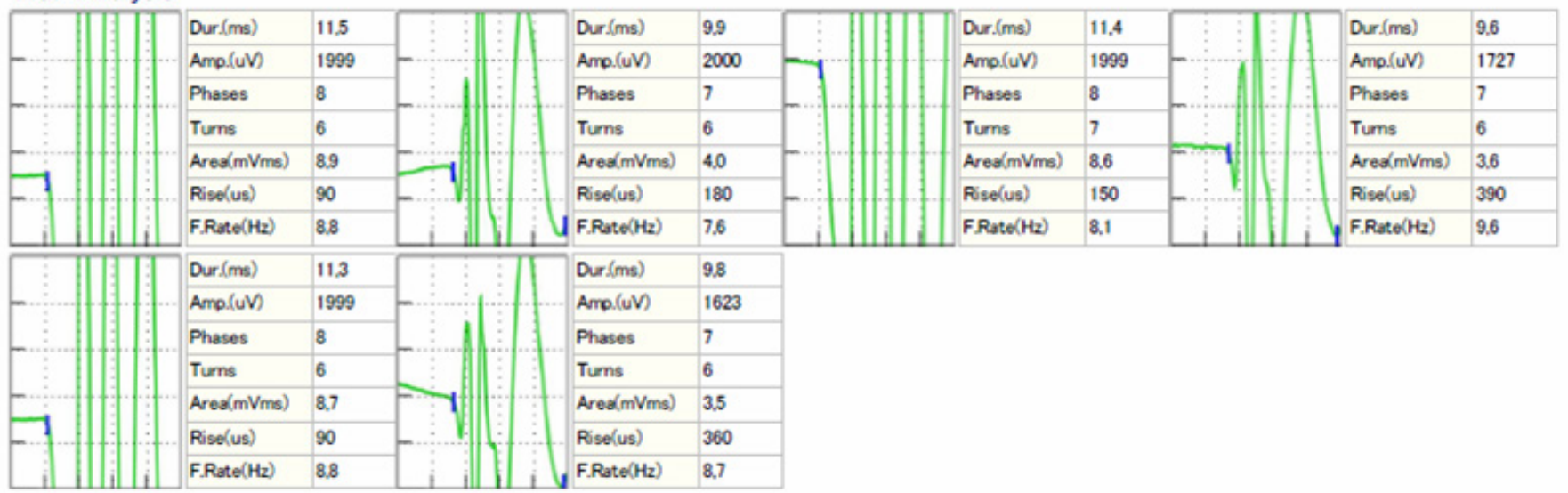

Fig. 3. EMG examination. Motor unit potentials with neurogenic changes in vastus lateralis muscle.

An important feature of the disease is marked cerebellar atrophy, especially of the vermis, which becomes evident in the second decade of life in brain imaging exams. There is a poor correlation between the degree of cerebellar atrophy and the patient's symptomatology. Despite severe cerebellar atrophy, our patient was independent. $(1,10,11)$

During the course of the disease, extrapyramidal signs may also occur, in some cases manifesting as progressive generalized dystonia, usually associated with cognitive decline. In our case extrapyramidal syndrome is manifested by cogwheel sign in the upper extremities, postural tremor of the extremities, but the cognitive functions are normal. (1)

Our case emphasize the emergence of protean clinical characteristics over many years and accentuate the need to consider late-onset TSD in the differential diagnosis of progressive muscular atrophy and early onset amyotrophic lateral sclerosis and even primary lateral sclerosis $(8,9,12)$

A study conducted by Gustavo H.B. Maegawa et al. had some promising results. It showed that pyrimethamine can be used as a potential pharmacological chaperone in late-onset TSD as it intensify the activity of the remaining HexA levels. (4) Another study, performed by Osher E et al demonstrated that this increase is transient and has no benefit on neurological and psychiatric symptoms.(3)

Even if there is still no effective treatment for late-onset TSD, early accurate diagnosis is particularly important because certain drugs, especially neuroleptics, can aggravate the symptoms and should be avoided in these patients. Also a late diagnosis may delay proper genetic counseling regarding disease progression and reproductive risks, which is especially important in Ashkenazi Jewish patients because the disease is transmitted in autosomal recessive fashion. $(2,13)$

In patients showing signs of lower motor neuron involvement, cerebellar and pyramidal signs and marked cerebellar atrophy the late-onset TSD should be suspected, and the first step in establishing the diagnosis should be to determine the serum activity of HexA.

\section{Acknowledgment}

This study was supported by the internal research Grant of The University of Medicine and Pharmacy Targu Mures, Grant Number 18/2015

\section{Conflict of interest}

None to declare.

\section{References}

1. Neudorfer O, Kolodny EH - Late-onset Tay-Sachs disease. Isr Med Assoc J. 2004 Feb;6(2):107-11

2. Neudorfer O, Pastores GM, Zeng BJ, et al - Late-onset Tay-Sachs disease: phenotypic characterization and genotypic correlations in 21 affected patients. Genet Med. 2005 Feb;7(2):119-23.

3. Osher E, Fattal-Valevski A, Sagie L, et al- Effect of cyclic, low dose pyrimethamine treatment in patients with Late Onset Tay Sachs: an open label, extended pilot study. Orphanet J Rare Dis. 2015 Apr 17;10:45.

4. Maegawa GH, Tropak M, Buttner J, et al- Pyrimethamine as a potential pharmacological chaperone for late-onset forms of GM2 gangliosidosis. J Biol Chem. 2007 Mar 23;282(12):9150-61.

5. Matsuzawa F, Aikawa S, Sakuraba H, et al- Structural basis of the GM2 gangliosidosis B variant. J Hum Genet. 2003;48(11):582-9.

6. Navon R, Kolodny EH, Mitsumoto H, et al- Ashkenazi-Jewish and nonJewish adult GM2 gangliosidosis patients share a common genetic defect. Am J Hum Genet. 1990 Apr;46(4):817-21. 
7. Deik A, Saunders-Pullman R. Atypical presentation of late-onset TaySachs disease. Muscle Nerve. 2014 May;49(5):768-71. doi: 10.1002/ mus.24146. Epub 2014 Feb 24

8. Jamrozik Z, Lugowska A, Gołębiowski M, Królicki L, Mączewska J, Kuźma-Kozakiewicz M. Late onset GM2 gangliosidosis mimicking spinal muscular atrophy. Gene. 2013 Sep 25;527(2):679-82. doi: 10.1016/j.gene.2013.06.030. Epub 2013 Jun 29.

9. Praline J, Guennoc AM, Vourc'h P, Sedel F, Andres CR, Corcia P. [Late onset Tay-Sachs disease may mimic adult SMA]. Rev Neurol (Paris). 2011 Jun-Jul;167(6-7):549-50. doi: 10.1016/j. neurol.2011.01.002. Epub 2011 Apr 8.

10. Steiner KM, Brenck J, Goericke S, Timmann D. Cerebellar atrophy and muscle weakness: late-onset Tay-Sachs disease outside Jewish populations. BMJ Case Rep. 2016 Mar 31;2016. pii: bcr2016214634. doi: 10.1136/bcr-2016-214634

11. Streifler JY, Gornish $M$, Hadar $\mathrm{H}$, et al- Brain imaging in late-onset GM2 gangliosidosis. Neurology. 1993 Oct;43(10):2055-8.

12. Godeiro-Junior C, Felicio AC, Benites V, Chieia MA, Oliveira AS.Lateonset hexosaminidase A deficiency mimicking primary lateral sclerosis. Arq Neuropsiquiatr. 2009 Mar;67(1):105-6.

13. Shapiro BE, Hatters-Friedman S, Fernandes-Filho JA, et al- Late-onset Tay-Sachs disease: adverse effects of medications and implications for treatment. Neurology. 2006 Sep 12;67(5):875-7. 